\title{
Intracranial fungal granuloma: a single-institute study of 90 cases over 18 years
}

\author{
Ajit Mishra, MCh, ${ }^{1}$ Andiperumal Raj Prabhuraj, MCh, ${ }^{1}$ Dhaval P. Shukla, MCh, ${ }^{1}$ \\ Bevinahalli N. Nandeesh, MD, ${ }^{2}$ Nagarathna Chandrashekar, MD, ${ }^{3}$ Arvinda Ramalingaiah, DM, ${ }^{4}$ \\ Arimappamagan Arivazhagan, $\mathrm{MCh},{ }^{1}$ Dhananjaya Ishwar Bhat, $\mathrm{MCh},{ }^{1}$ Sampath Somanna, $\mathrm{MCh},{ }^{1}$ \\ and Bhagavatula Indira Devi, $\mathrm{MCh}^{1}$
}

Departments of ${ }^{1}$ Neurosurgery, ${ }^{2}$ Neuropathology, ${ }^{3}$ Neuromicrobiology, and ${ }^{4}$ Neuroimaging and Interventional Radiology, National Institute of Mental Health and Neurosciences, Bengaluru, Karnataka, India

OBJECTIVE Intracranial fungal granuloma (IFG) remains an uncommon entity. The authors report a single-institute study of 90 cases of IFG, which is the largest study until now.

METHODS In this retrospective study, all cases of IFG surgically treated in the years 2001-2018 were included. Data were obtained from the medical records and the pathology, microbiology, and radiology departments. All relevant clinical data, imaging characteristics, surgical procedure performed, perioperative findings, and follow-up data were recorded from the case files. Telephonic follow-up was also performed for a few patients to find out their current status.

RESULTS A total of 90 cases consisting of 64 males $(71.1 \%)$ and 26 (28.9\%) females were evaluated. The mean patient age was 40.2 years (range 1-79 years). Headache (54 patients) was the most common presenting complaint, followed by visual symptoms (35 patients), fever (21 patients), and others such as limb weakness (13 patients) or seizure ( 9 patients). Cranial nerve involvement was the most common sign (47 patients), followed by motor deficit (22 patients) and papilledema (7 patients). The mean duration of symptoms before presentation was 6.4 months (range $0.06-48$ months). Thirty patients (33.3\%) had predisposing factors like diabetes mellitus, tuberculosis, or other immunocompromised status. A pure intracranial location of the IFG was seen in 49 cases $(54.4 \%)$, whereas rhinocerebral or paranasal sinus involvement was seen in 41 cases (45.6\%). Open surgery, that is, craniotomy and decompression, was performed in 55 cases, endoscopic biopsy was done in 30 cases, and stereotactic biopsy was performed in 5 cases. Aspergilloma (43 patients) was the most common fungal mass, followed by zygomycosis (13 patients), chromomycosis (9 patients), cryptococcoma (7 patients), mucormycosis (5 patients), and candida infection (1 patient). In 12 cases, the exact fungal phenotype could not be identified. Follow-up was available for $69 / 90$ patients $(76.7 \%)$. The mean duration of the follow-up was 37.97 months (range 3-144 months). The mortality rate was $52.2 \%$ (36/69 patients) among the patients with available follow-up.

CONCLUSIONS A high index of suspicion for IFG should exist for patients with an immunocompromised status and diabetic patients with rhinocerebral mass lesions. Early diagnosis, aggressive surgical decompression, and a course of promptly initiated antifungal therapy are associated with a better prognosis.

https://thejns.org/doi/abs/10.3171/2019.5.FOCUS19252

KEYWORDS intracranial; fungal granuloma; immunocompromised; immunocompetent

$\mathrm{F}$ UNGAL infections of the central nervous system are rare. However, the incidence and diagnosis of these disease entities are increasing,, 93 mostly because of better diagnostic modalities such as imaging studies, microbiological tests, and pathology tests. Etiological factors contributing to the increasing incidence include immunocompromised states such as diabetes mellitus, renal failure, malnutrition, acquired immunodeficiency syndrome, lymphoproliferative malignancies, and neutropenia, as well as the use of broad-spectrum antibiotics. Other possible factors are longer life expectancy, larger proportion of an aging population, widespread use of immunosuppressive drugs, longer survival of immunocompromised patients, increasing numbers of human immunodeficiency virus (HIV) infections, and poor nutritional status. ${ }^{2,18,38}$ These infections can involve the meninges, calvaria, brain,

ABBREVIATIONS ACF = anterior cranial fossa; $A M B=$ amphotericin B; HIV = human immunodeficiency virus; IFG = intracranial fungal granuloma; PNS = paranasal sinus . SUBMITTED March 18, 2019. ACCEPTED May 10, 2019

INCLUDE WHEN CITING DOI: 10.3171/2019.5.FOCUS19252. 
and intracranial vessels in different forms, severity, and combinations. ${ }^{4,7,22,30}$

Fungal infections pose a diagnostic challenge to clinicians and radiologists because of their resemblance to a pyogenic abscess, benign lesion, or even malignancy. They have high rates of death and morbidity, but early diagnosis and prompt treatment with surgery and antifungal drugs may cure a significant proportion of patients.

Although fungal infections themselves are relatively frequent, actual granulomatous masses due to fungal infections are rare. The rarity of the disease even in a highvolume center can be seen from the sparsity of the literature on this topic. In the last 2 decades, only six large series from India have described fungal granulomas. ${ }^{9,23,24,31,32,36}$ Here, we report our experience with fungal granulomas surgically managed over the last 18 years at our institute. Detailed clinical, radiological, surgical, pathological, and microbiological characteristics are described.

\section{Methods}

This retrospective study from a single institute (National Institute of Mental Health and Neurosciences, Bangalore, India) was focused on all cases of intracranial fungal granuloma (IFG) surgically treated in an 18-year period (2001-2018). Case files were taken from the medical record section. Relevant imaging details from CT or MRI of the brain, chest radiography, or paranasal sinus (PNS) CT were obtained from the radiology department. In a few cases, imaging details were not available in the radiology department; in such cases imaging findings mentioned in case sheets were used for our analysis. Additional data regarding fungal morphology and phenotype were also obtained from our neuropathology department. Culture reports from the Department of Neuromicrobiology at our institute were available in $60 \%$ of the cases. In some cases, even during the surgery, fungal granuloma was not kept in the differential diagnosis; hence, biopsy material could not be sent for culture. Other tests such as blood test and urine culture in almost all cases, sputum culture in those having tuberculosis, and CD4 counts in HIVinfected cases were performed. After surgical treatment, all patients were started on antifungal drugs based on the fungal morphology. Amphotericin B (AMB) was used most frequently. Based on final histopathology, different antifungal drugs such as fluconazole, flucytosine, or voriconazole were used in different combinations. Intravenous route delivery was used for a minimum of 6 weeks, and then depending on the culture report, it was converted to oral medications. All the relevant clinical data such as age, sex, signs and symptoms, symptom duration, predisposing factors, location of the disease with special attention to the PNS, surgical procedure performed, intraoperative findings, and follow-up data were recorded from the case files. For a few patients, no follow-up information was available in the case file, so in those cases, patients were contacted via telephone to find out about their current status.

\section{Results}

\section{Clinical Presentation}

A total of 90 cases of IFGs were studied. The mean pa-
TABLE 1. General demographic details and outcomes of 90 patients with IFGs

\begin{tabular}{lc}
\hline \multicolumn{1}{c}{ Variable } & Value \\
\hline Mean age in yrs & $40.2 \pm 15.97(1-79)$ \\
\hline Sex & $64(71.1 \%)$ \\
$\quad$ Male & $26(28.9 \%)$ \\
$\quad$ Female & $6.36 \pm 9.22(0.06-48)^{*}$ \\
\hline Mean symptom duration in mos & $69 / 90(76.7 \%)$ \\
\hline Patients w/ FU & $37.97 \pm 43.18(3-144)$ \\
\hline FU period in mos in $69 / 90$ patients & $36 / 69(52.2 \%)$ \\
\hline Deaths among those w/ FU & \\
\hline FU = follow-up. & \\
Values are expressed as the mean \pm standard deviation (range) or as fre- \\
quency (\%). \\
* Two days to 48 months.
\end{tabular}

tient age was 40.2 years (range 1-79 years), and there were $64(71.1 \%)$ males and $26(28.9 \%)$ females (Table 1). The mean duration of symptoms before presentation to the hospital was 6.36 months (range 0.06-48 months). Headache (54 patients) was the most common presenting complaint, followed by visual symptoms (35 patients), fever (21 patients), limb weakness (13 patients), and seizure (9 patients; Table 2). Cranial nerve involvement was the most common sign (47 patients), followed by motor deficit (22 patients) and papilledema (7 patients). Ocular motor nerves (cranial nerves III, IV, and VI) and optic nerves were most frequently involved most likely because of the close relation of the orbital apex/cavernous sinus to the PNSs. Hemiparesis was seen in 22 patients because of either direct mass effect from the lesion or its associated perilesional edema over the motor cortex, basal ganglia, or internal capsule.

\section{Predisposing Factors}

Of the 90 patients, 30 (33.3\%) had predisposing factors like diabetes mellitus, tuberculosis, trauma/direct inoculation, contiguous (mastoid) or remote (infective endocarditis) infection, or other immunocompromised status like HIV infection, neutropenia, Guillain-Barré syndrome, or a post-chemoradiation therapy status (Table 3). Direct inoculation in our series implies the development of IFG after a traumatic compound skull fracture.

\section{Location of the IFG}

A pure intracranial location of the IFG was seen in 49/90 cases (54.4\%), whereas rhinocerebral or PNS involvement was seen in $41 / 90$ cases (45.6\%; Table 4). IFG was seen as a single lesion in 75 cases $(83.3 \%)$ and in multiples in 15 cases $(16.7 \%)$. The frontal lobe was most frequently involved either in isolation or in most cases as an extension from the sphenoid sinus, orbital region, or anterior cranial fossa (ACF) base. Cavernous sinus and parasellar region involvement were frequently seen. Pure cerebellar involvement was seen in 5 cases and the thalamus in 3 cases.

\section{Imaging}

CT was used as the sole modality for diagnosis in 55 
TABLE 2. Presenting clinical symptoms and signs of 90 patients with IFGs

\begin{tabular}{cc}
\hline & No. $(\%)$ \\
\hline Symptom & \\
\hline Headache & $54(60 \%)$ \\
\hline Visual disturbance & $35(38.9 \%)$ \\
\hline Fever & $21(23.3 \%)$ \\
\hline Limb weakness & $13(14.4 \%)$ \\
\hline Seizure & $9(10 \%)$ \\
\hline Altered sensorium & $6(6.7 \%)$ \\
\hline Nasal discharge & $2(2.2 \%)$ \\
\hline Loss of smell & $1(1.1 \%)$ \\
\hline Wound discharge & $1(1.1 \%)$ \\
\hline Hearing loss & $1(1.1 \%)$ \\
\hline Sign & \\
\hline Cranial nerve involvement & $47(52.2 \%)$ \\
\hline Motor deficit & $22(24.4 \%)$ \\
\hline Papilledema & $7(7.8 \%)$ \\
\hline Cerebellar signs & $6(6.7 \%)$ \\
\hline Meningismus & $3(3.3 \%)$ \\
\hline Anosmia & $2(2.2 \%)$ \\
\hline Aphasia & $2(2.2 \%)$ \\
\hline Behavioral changes & $1(1.1 \%)$ \\
\hline Periorbital edema & $1(1.1 \%)$ \\
\hline
\end{tabular}

cases, whereas MRI was available in 35 cases. We had seen two different patterns of IFG on CT. The first pattern was a predominantly hyperdense lesion on plain CT (36 patients) with surrounding edema (hypodense), and the second pattern was a hyperdense periphery with a hypodense central part of the lesion along with perilesional edema (19 patients). Bony destruction was seen if the lesion involved the skull base. The enhancement pattern was either uniform or a peripheral ring pattern. On MRI, the most common finding of IFG was T1 hypointense/isointense and $\mathrm{T} 2$ hypointense with heterogeneous contrast enhancement (22 patients; Fig. 1). Less commonly (13 patients), the lesion was hypointense/isointense on T1-weighted images and hyperintense on T2-weighted images with an occasional peripheral rim of hypointensity. Magnetic resonance spectroscopy was done in 10 cases, and all scans showed an increased lipid-lactate peak with a reduction in other metabolites. Diffusion-weighted imaging was available in 8 cases, all of which showed diffusion restriction. In 33 patients (36.7\%), IFG was not the working diagnosis. In the remaining 57 patients (63.3\%), even though IFG was not diagnosed based on clinical or imaging features, it was kept as one of the main differential diagnoses.

\section{Treatment}

Most of the patients underwent open surgery, i.e., craniotomy and excision (55 cases). Endoscopic biopsy (transnasal with or without transsphenoidal approach) was performed in 30 cases, and 5 patients underwent stereotactic biopsy. Three patients required a ventriculoperitoneal
TABLE 3. Predisposing factors of patients with IFGs

\begin{tabular}{lc}
\hline \multicolumn{1}{c}{ Factor } & No. \\
\hline DM & 16 \\
\hline TB & 4 \\
\hline Trauma/direct inoculation & 2 \\
\hline HIV & 2 \\
\hline Infection (contiguous/remote) & \\
CSOM & 1 \\
Infective endocarditis & 1 \\
Immunocompromised & \\
CD4 count <200 cells/mm & \\
Neutropenia & 1 \\
GBS & 1 \\
Post-chemoradiation & 1 \\
\hline
\end{tabular}

$\mathrm{CSOM}=$ chronic suppurative otitis media; $\mathrm{DM}=$ diabetes mellitus; $\mathrm{GBS}=$ Guillain-Barré syndrome; TB = tuberculosis.

shunt for obstructive hydrocephalus. A solid/firm lesion was seen during surgery in 71 cases (78.9\%), whereas 19 cases $(21.1 \%)$ had an abscess.

\section{Pathogen}

Aspergilloma (43 patients) was the most common fungal lesion based on histopathological diagnosis, followed by zygomycosis (13 patients), chromomycosis (9 patients), cryptococcoma (7 patients), mucormycosis (5 patients), and candida infection (1 patient). Figure 2 shows the various histopathological appearances along with special stains of the fungal pathogens seen in the current study. In 12 of our cases, the exact fungal phenotype could not be identified based on histopathology (Table 5). Fungal cul-

TABLE 4. Location of the fungal lesion among 90 patients with IFGs

\begin{tabular}{cc}
\hline \multicolumn{1}{c}{ Location } & No. \\
\hline Pure intracranial & $49(54.4 \%)$ \\
Intraaxial & 39 \\
Frontal & 17 \\
Parietooccipital & 7 \\
Temporal & 7 \\
Cerebellar & 5 \\
Thalamus & 3 \\
Extraaxial & 10 \\
ACF & 6 \\
MCF & 3 \\
ACF \& MCF & 1 \\
Sinonasal or rhinocerebral & $41(45.6 \%)$ \\
ACF w/ orbit & 10 \\
Cavernous sinus \& parasellar region & 11 \\
ACF & 9 \\
MCF & 5 \\
ACF \& MCF & 2 \\
MCF \& PCF & 2 \\
ACF w/ parenchyma & 2 \\
\hline
\end{tabular}

$\mathrm{MCF}=$ middle cranial fossa; $\mathrm{PCF}=$ posterior cranial fossa. 

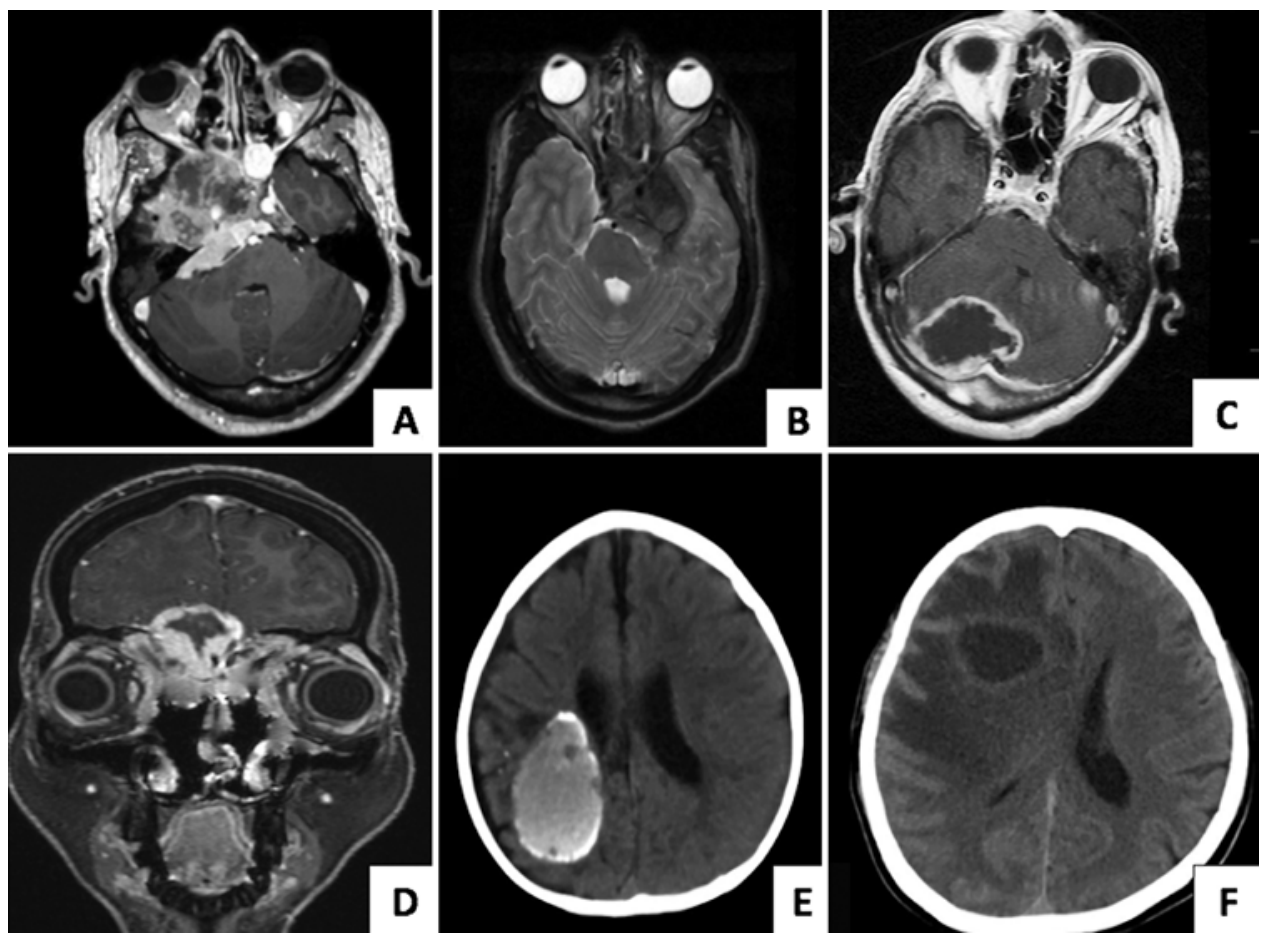

FIG. 1. Neuroimaging of the various fungal lesions. A: Axial T1-weighted contrast MRI shows a heterogeneously enhancing lesion with areas of central necrosis in the right temporal base extending to the posterior fossa and also involving the ethmoid sinus (aspergilloma). B: Axial T2-weighted MRI shows a hypointense lesion in the left medial temporal region with involvement of the cavernous sinus and the ethmoid sinus (aspergilloma). C: Axial T1-weighted contrast MRI shows a peripheral enhancing lesion with a central necrotic part in the right cerebellar hemisphere (cryptococcoma). D: Coronal T1-weighted contrast MRI shows a heterogeneously enhancing lesion in the ACF base with involvement of the PNS (aspergilloma). E: Axial CT shows a well-defined hyperdense lesion in the right parietal region with a few specks of calcification at the rim (chromoblastomycosis). F: Axial CT shows a well-defined hypodense lesion in the right frontal region with a hyperdense rim and perilesional edema (chromoblastomycosis).

ture reports were available in only $60 \%$ of the cases $(\mathrm{n}=$ 54). In some cases, even during surgery, fungal granuloma was not kept in the differential diagnosis; in those cases, the biopsy material could not be sent for culture. Thirtyseven of the 54 cultures were positive for the fungal pathogen, and 17 cultures had no growth (Table 6).

\section{Antifungal Agents and Outcome}

Antifungal medication, mainly AMB, voriconazole, fluconazole, or flucytosine as monotherapy or in combination, was started immediately once the diagnosis of fungal infection was considered or sometimes after the biopsy report. It was given for a minimum of 6 weeks via an intravenous route and was then converted to oral form. Many patients required medications up to 18 months depending on the response to treatment based on clinical or radiological improvement.

We had a few in-hospital deaths (12 patients) because of disease progression or drug toxicity, mainly due to renal failure. Follow-up was available for $69 / 90$ patients (76.7\%). The mean duration of the follow-up was 37.97 months (range 3-144 months). The mortality rate was $52.2 \%$ (36/69 patients) among the patients with available follow-up. Of the 36 deaths, 12 occurred within a week of surgery, 6 within 1 month, 10 within 3 months, 5 within 6 months, and 3 within 2 years. Cumulative mortality at different points after surgery was as follows: $17.4 \%$ at 1 week, $26.1 \%$ at 1 month, $40.6 \%$ at 3 months, $47.8 \%$ at 6 months, and $52.2 \%$ at 2 years. Eighteen of 36 deaths had predisposing factors that led to a fatal outcome.

\section{Discussion}

Fungal infections of the central nervous system may take the form of an acute, rapidly fatal illness with meningitis, encephalitis, vasculitis, and abscess formation or a chronic form with granuloma formation. ${ }^{32}$ Our study was limited to patients with histopathologically proven fungal granulomas.

\section{Predisposing Factors}

IFGs in immunocompetent patients have been predominantly reported from India, Pakistan, Saudi Arabia, Africa, and California in the United States. ${ }^{15,35}$ It is postulated that a hot, dry climate with a high content of Aspergillus spores in the atmosphere due to agricultural dust is a probable cause of IFGs in persons residing in these regions. Nearly $50 \%$ of patients with IFGs have no overt predisposing illness or evidence of immunosuppression., ${ }^{9,14,20,32,35}$ It is possible that in some of these patients, there may be subclinical impairment of cell-mediated immunity. ${ }^{28}$ Rarely, fungal infection may follow direct inoculation of the brain during intracranial or transsphenoidal surgery or following trauma. ${ }^{1,33}$ We had 2 such cases in which the patient 


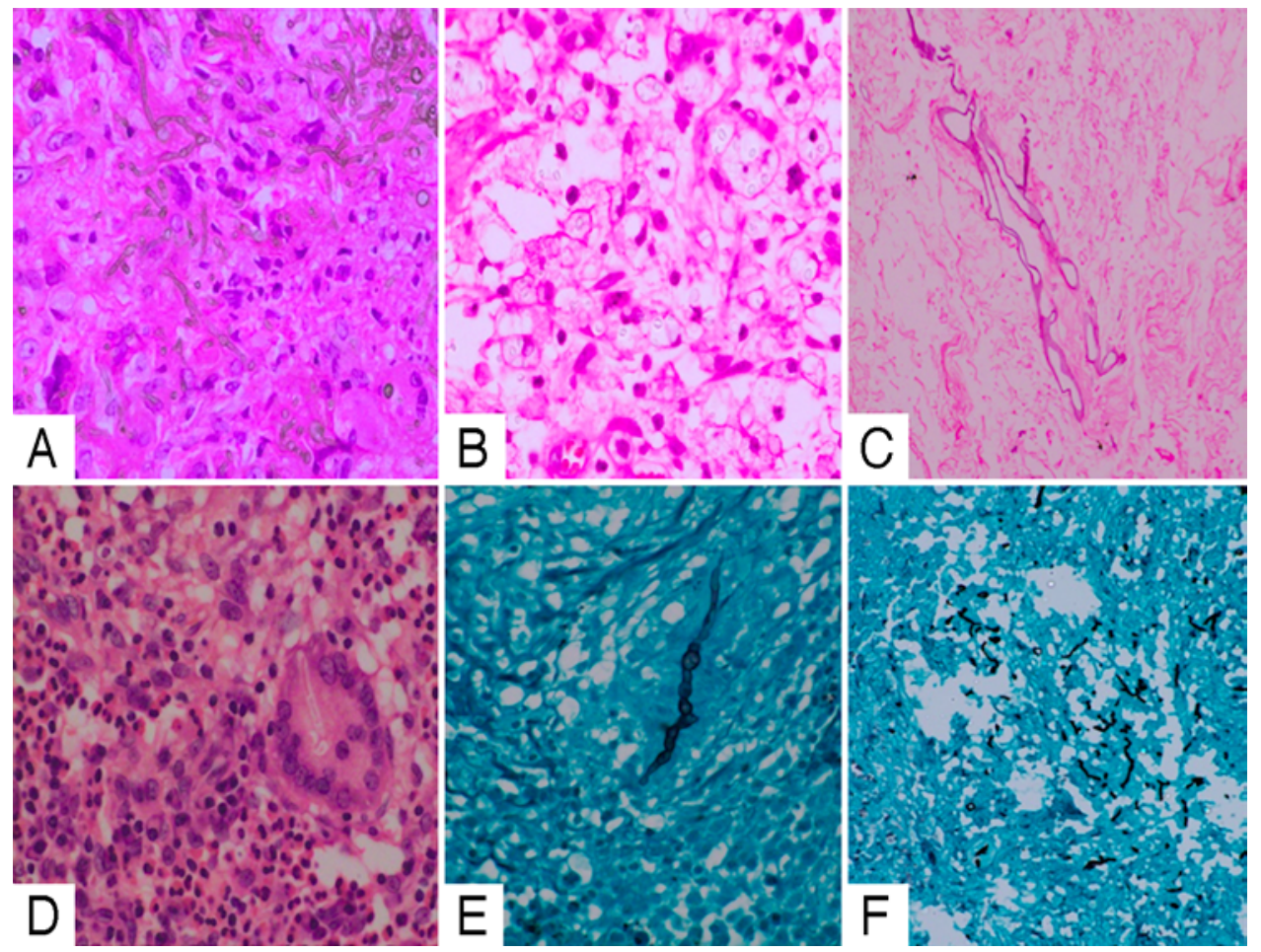

FIG. 2. Histological and immunohistochemistry images of various fungal lesions. A: Photomicrograph showing necrotizing granulomatous inflammation with pigmented fungal hyphae (phaeohyphomycosis, chromoblastomycosis). H \& E. B: Photomicrograph showing necrotizing inflammation with yeast structures of Cryptococcus. H \& E. C: Mucormycosis: broad aseptate hyphae branching at right angles. Periodic acid-Schiff. D: Aspergillus: granuloma with fungal hyphae within the giant cell. H \& E. E: Aspergillus: slender septate hyphae branching at acute angles. Gomori methenamine silver. F: Granulomatous inflammation with pigmented fungal hyphae (phaeohyphomycosis, chromoblastomycosis). Gomori methenamine silver. Original magnification $\times 200(\mathrm{~A}-\mathrm{E})$ and $\times 100(\mathrm{~F})$.

presented to us with IFG after a definitive history of compound fracture of the skull vault.

IFGs usually develop by the spread of fungal infection to the intracranial compartment from a systemic source of infection. The disease usually spreads through the hematogenous route, but invasion of contiguous tissues and spread into the intracranial compartment is also common in the case of PNS and mastoid infections. However, in a proportion of patients with IFG, no obvious systemic source for the fungal infection is discernible despite an extensive search. ${ }^{28}$ We had only 2 cases in which there was evidence of systemic infection. One case had mastoid infection and the other had infective endocarditis.

A previous study from our institute showed that $68 \%$ of patients had some kind of predisposing factor. ${ }^{9}$ In the current study, however, only $33.3 \%$ had such factors, which again indicates that the incidence of IFG is on the rise even in immunocompetent patients. In a large study from India by Naik et al., most of the 66 patients did not have any predisposing factors. ${ }^{24}$

\section{Clinical Presentation}

The mean age of our patients was 40.2 years, which is similar to the mean age in a previous study from our institute $^{9}$ and comparable to 32.3 years and 28 years in the studies by Naik et al. ${ }^{24}$ and Sharma et al., ${ }^{32}$ respectively. The greater IFG incidence in males in our study is probably the result of their increased exposure to the outside environment. This finding has also been suggested by other authors. ${ }^{21,23,27,32}$

Most of our patients presented with headache (54 patients) and cranial nerve symptoms (47 patients), mainly the optic nerve and ocular motor nerves. Seizure was a rare presenting symptom and was observed in only 9 of our cases. These findings are consistent with data in other studies. ${ }^{9,24,32}$ Fever was seen in 21 of our patients. In the literature, there are also reports of patients who present with an increased incidence of stroke. ${ }^{12,14}$ Subarachnoid hem-

TABLE 5. Fungal phenotype based on histopathology*

\begin{tabular}{lrc}
\hline \multicolumn{1}{c}{ Type } & No. & $\%$ \\
\hline Aspergilloma & 43 & 47.8 \\
\hline Cryptococcoma & 7 & 7.8 \\
\hline Chromomycosis & 9 & 10 \\
\hline Zygomycosis & 13 & 14.4 \\
\hline Mucormycosis & 5 & 5.6 \\
\hline Candida & 1 & 1.1 \\
\hline Fungal & 12 & 13.3 \\
\hline Total & 90 & 100 \\
\hline
\end{tabular}

${ }^{*}$ Not culture proven. 
TABLE 6. Culture-proven fungal pathogen

\begin{tabular}{lc}
\hline \multicolumn{1}{c}{ Pathogen } & No. \\
\hline Aspergillus fumigatus & 7 \\
\hline Aspergillus flavus & 4 \\
\hline Aspergillus spp. & 4 \\
\hline Cryptococcus neoformans & 5 \\
\hline Scytalidium dimidiatum & 1 \\
\hline Septate fungi & 1 \\
\hline Cladosporium spp. & 1 \\
\hline Fungus & 4 \\
\hline Cladophialophora bantiana & 6 \\
\hline Mucor spp. & 1 \\
\hline Candida albicans & 1 \\
\hline Fonsecaea pedrosoi & 1 \\
\hline Cryptococcus gattii & 1 \\
\hline No growth & $37(68.5 \%)$ \\
\hline Total positive cultures & $54 / 90(60 \%)$ \\
\hline Total cultures available &
\end{tabular}

orrhage (SAH) and mycotic aneurysm formation are rare presentations of IFG. ${ }^{3,23,24,32}$ However, none of our patients presented with stroke or SAH.

\section{Location of the Granuloma}

As seen in other studies, the frontal lobe, followed by the temporal lobe, was the most common site for IFG. ${ }^{9,24,32} \mathrm{~A}$ possible explanation would be the close relation to the PNS and ACF base. Isolated frontal IFGs have also been seen without contiguous sinus involvement. The sella, parasellar region, and cavernous sinus with the middle cranial fossa base were other common locations seen in our study (Table 4). Posterior cranial fossa involvement was rare, and we had only 5 isolated cerebellar lesions. The route of spread for the pure intraparenchymal variant has been proposed to be hematogenous or even retrograde thrombophlebitis, especially in the case of cerebellar IFGs. Selvam et al. have a detailed staging system for invasive rhinocerebral fungal granuloma according to the extent of involvement and the final outcome. ${ }^{31}$

\section{Imaging Features and Differentials}

In his review article on IFG, Rajshekhar has explained how it can be missed from the list of differential diagnoses. ${ }^{28}$ According to him, the caseload of IFGs would not exceed two per year even in large neurosurgical centers in India. Surgery for IFGs would constitute about one per 1000 neurosurgical procedures performed at these centers. With these prevalence rates, it is not surprising that IFGs are rarely suspected in patients with isolated intracranial masses and are misdiagnosed as glioma, tuberculoma, lymphoma, meningioma, or cholesteatoma.

Several reports deal with imaging finding of IFGs. ${ }^{8-}$ $10,16,17,25,32,34$ Irregular isodense/hyperdense lesions with faint heterogeneous contrast enhancement on CT scan and perilesional edema are features of fungal lesions. ${ }^{10,13,25,26,34}$ Evidence of PNS involvement, lesions close to the PNS, small areas of bone destruction, and infarcts caused by arteritis favor a fungal etiology. $5,6,10,11,17,24,25,33$ Neuroimaging patterns that have been reported vary depending on the immunological status of the patient and the age of the lesions. ${ }^{10,17,33}$ Saini et al. have described the characteristic imaging features of aspergilloma in immunocompetent patients. ${ }^{29}$ These authors concluded that hyperdense sinonasal disease with bone destruction and intracranial extension on CT, hypointense signal intensity of the lesions on T2-weighted MRI, areas of restricted diffusion, decreased perfusion on perfusion-weighted imaging, and hemorrhages are key to the imaging diagnosis of fungal infection. Dubey et al. have shown the distinguishing feature between cryptococcoma and aspergilloma based on MRI. ${ }^{9}$

The classic CT finding for IFG in our study was a predominantly hyperdense lesion on plain CT with peripheral edema or hyperdense periphery with a hypodense central portion of the lesion. Bone destruction was common in skull base lesions. The typical pattern on MRI was isointense/hypointense on T1-weighted imaging and hypointense on T2-weighted images. T2 hyperintense lesions have also been seen with a peripheral rim of hypointensity and heterogeneous contrast enhancement. Magnetic resonance spectroscopy and diffusion-weighted imaging definitely help the physician in the diagnosis of IFG. Besides imaging the brain with CT and MRI, a few patients may have abnormality diagnosed on chest radiograph or thorax $\mathrm{CT}$, especially in cases of aspergilloma. However, we did not find any such abnormal chest findings in our patients.

\section{Fungal Pathogens}

Aspergillus was the most common phenotype (47.8\%) causing IFG in the current study as well as in previous studies. ${ }^{9,24,32,36}$ Other phenotypes were cryptococcoma, mucormycosis, zygomycosis, and chromomycosis (Table $5)$. However, when we looked at the fungal culture, only $60 \%$ of the reports were available (Table 6). Aspergillus with different subspecies remains the most common pathogen. Cladophialophora bantiana was seen in 6 patients, Cryptococcus neoformans in 5 patients, Cryptococcus gattii in 1 patient, and Fonsecaea pedrosoi in 1 case. All of the above pathogens have been reported from our institute as part of a case series or case report by Garg et al., ${ }^{11}$ Uppar et al., ${ }^{37}$ and Madhugiri et al. ${ }^{19}$

\section{Treatment}

Aggressive surgical excision followed by the timely administration of antifungal drugs is the cornerstone of the management of IFGs. Of the 90 patients in our study, 55 required open surgery in the form of craniotomy and excision of the granuloma. We tried to excise the granuloma completely; however, in a few places, especially in the skull base region, because of a lesion's proximity or adherence to a vital neurovascular structure, we were restricted in performing an aggressive resection. In 30 patients in whom the lesion was accessible via an endoscopic transnasal approach, endoscopic biopsy was performed, followed by the administration of antifungal drugs. In 5 cases in which the lesion was small, involving an eloquent region of the brain, and without any significant mass effect or perilesional edema, we performed stereotactic biopsy. 
Three patients required CSF diversion in the form of a ventriculoperitoneal shunt for obstructive hydrocephalus.

\section{Outcome}

The final outcome of IFG remains poor; however, results are getting better with early diagnosis because of neuroimaging, increased awareness of the patient as well as the treating physician, aggressive resection, and better antifungal drugs.

If we look at mortality data from the year 1985, we note a gradual decline to the present. The initial study by Yanai et al..$^{39}$ in 1985 had a mortality rate of more than $95 \%$. In 1996, Sharma et al. ${ }^{32}$ showed a mortality rate of around $50 \%$. Similar rates of mortality have been documented: $63 \%$ by Dubey et al. ${ }^{9}$ in $2005,62.8 \%$ by Young et al. ${ }^{40}$ in 1985 , and $36.4 \%$ by Naik et al..$^{24}$ in 2015 . One study by Siddiqui et al. ${ }^{34}$ has shown quite a low rate of mortality at $15 \%$. Our study had a mortality rate of $52.2 \%$, which is similar to rates in other major series.

We started the antifungal agents as soon as the working diagnosis of IFG was made based on clinical and imaging features. Very occasionally, we had the experience of overtreatment in some cases. In one case, a young boy had the classic presentation of the disease in the right basifrontal region extending into the frontal air sinus. The patient's history and imaging features all favored a diagnosis of fungal granuloma. The patient was started on empirical AMB and taken for surgery. Antifungal medication was continued for a week postoperatively until the definitive biopsy report came, and to the surprise of everyone, it turned out to be lymphoma. It is better to keep the diagnosis of fungal granuloma in mind and start the treatment early rather than waiting for the definitive biopsy or culture report, which takes a minimum of 5-7 days and deprives the patient of the beneficial or potentially curative effect of antifungal medications.

\section{Study Limitations}

This was a retrospective study from a single institute. In terms of follow-up, telephonic conversations were used for a few patients, and the exact cause of death could not be established for those deaths occurring outside the hospital. So, it was difficult to predict whether death was attributable to fungal disease per se, antifungal toxicity, or comorbidity.

\section{Conclusions}

A high index of suspicion for IFG should exist for patients with an immunocompromised status or diabetic patients with rhinocerebral mass lesions. Early diagnosis, aggressive surgical excision, and a course of promptly initiated antifungal therapy are associated with a better prognosis. Mortality is high for immunocompromised patients even after extensive surgery and antifungal treatment.

\section{References}

1. Ahuja GK, Jain N, Vijayaraghavan M, Roy S: Cerebral mycotic aneurysm of fungal origin. Case report. J Neurosurg 49:107-110, 1978

2. Aisner J, Murillo J, Schimpff SC, Steere AC: Invasive asper- gillosis in acute leukemia: correlation with nose cultures and antibiotic use. Ann Intern Med 90:4-9, 1979

3. Banerjee AK, Singh MS, Kak VK, Talwar P, Rout D: Cerebral aspergillosis report of 8 cases. Indian J Pathol Microbiol 20:91-99, 1977

4. Beal MF, O'Carroll CP, Kleinman GM, Grossman RI: Aspergillosis of the nervous system. Neurology 32:473-479, 1982

5. Bergstrom L, Hemenway WG, Barnhart RA: Rhinocerebral and otologic mucormycosis. Ann Otol Rhinol Laryngol 79:70-81, 1970

6. Chakrabarti A: Epidemiology of central nervous system mycoses. Neurol India 55:191-197, 2007

7. Deshpande DH, Desai AP, Dastur HM: Aspergillosis of the central nervous system. A clinical and mycopathological study of 9 cases. Neurol India 23:167-175, 1975

8. Dortzbach RK, Segrest DR: Orbital aspergillosis. Ophthalmic Surg 14:240-244, 1983

9. Dubey A, Patwardhan RV, Sampth S, Santosh V, Kolluri S, Nanda A: Intracranial fungal granuloma: analysis of 40 patients and review of the literature. Surg Neurol 63:254-260, 2005

10. Gabelmann A, Klein S, Kern W, Krüger S, Brambs HJ, Rieber-Brambs A, et al: Relevant imaging findings of cerebral aspergillosis on MRI: a retrospective case-based study in immunocompromised patients. Eur J Neurol 14:548-555, 2007

11. Garg N, Devi IB, Vajramani GV, Nagarathna S, Sampath S, Chandramouli BA, et al: Central nervous system cladosporiosis: an account of ten culture-proven cases. Neurol India 55:282-288, 2007

12. Goldstein LS, Haug MT III, Perl J II, Perl MK, Maurer JR, Arroliga AC, et al: Central nervous system complications after lung transplantation. J Heart Lung Transplant 17:185-191, 1998

13. Grossman RI, Davis KR, Taveras JM, Beal MF, O'Carroll $\mathrm{CP}$ : Computed tomography of intracranial aspergillosis. J Comput Assist Tomogr 5:646-650, 1981

14. Haran RP, Chandy MJ: Intracranial aspergillus granuloma. Br J Neurosurg 7:383-388, 1993

15. Jamjoom AB, al-Hedaithy SAS, Jamjoom ZAB, al-Hedaithy M, el-Watidy SF, Rahman N, et al: Intracranial mycotic infections in neurosurgical practice. Acta Neurochir (Wien) 137:78-84, 1995

16. Kanaly CW, Selznick LA, Cummings TJ, Adamson DC: Cerebellar cryptococcoma in a patient with undiagnosed sarcoidosis: case report. Neurosurgery 60:E571, 2007

17. Keyik B, Edgüer T, Hekimoğlu B: Conventional and diffusion-weighted MR imaging of cerebral aspergillosis. Diagn Interv Radiol 11:199-201, 2005

18. Levy RM, Bredesen DE, Rosenblum ML: Neurological manifestations of the acquired immunodeficiency syndrome (AIDS): experience at UCSF and review of the literature. J Neurosurg 62:475-495, 1985

19. Madhugiri VS, Bhagavatula ID, Mahadevan A, Siddaiah N: An unusual infection, an unusual outcome-Fonsecaea pedrosoi cerebral granuloma. J Neurosurg Pediatr 8:229-232, 2011

20. Marcinkowski M, Bauer K, Stoltenburg-Didinger G, Versmold H: Fungal brain abscesses in neonates: Sonographic appearances and corresponding histopathologic findings. J Clin Ultrasound 29:417-421, 2001

21. McGill TJ, Simpson G, Healy GB: Fulminant aspergillosis of the nose and paranasal sinuses: a new clinical entity. Laryngoscope 90:748-754, 1980

22. Mohandas S, Ahuja GK, Sood VP, Virmani V: Aspergillosis of the central nervous system. J Neurol Sci 38:229-233, 1978

23. Murthy JM, Sundaram C, Prasad VS, Purohit AK, Rammurti S, Laxmi V: Aspergillosis of central nervous system: a study 
of 21 patients seen in a university hospital in south India. $\mathbf{J}$ Assoc Physicians India 48:677-681, 2000

24. Naik V, Ahmed FU, Gupta A, Garg A, Sarkar C, Sharma B, et al: Intracranial fungal granulomas: a single institutional clinicopathologic study of 66 patients and review of the literature. World Neurosurg 83:1166-1172, 2015

25. Okafuji T, Yabuuchi H, Nagatoshi Y, Hattanda Y, Fukuya T: CT and MR findings of brain aspergillosis. Comput Med Imaging Graph 27:489-492, 2003

26. Oner AY, Celik H, Akpek S, Tokgoz N: Central nervous system aspergillosis: magnetic resonance imaging, diffusionweighted imaging, and magnetic resonance spectroscopy features. Acta Radiol 47:408-412, 2006

27. Parker JC Jr, McCloskey JJ, Lee RS: The emergence of candidosis. The dominant postmortem cerebral mycosis. Am J Clin Pathol 70:31-36, 1978

28. Rajshekhar V: Surgical management of intracranial fungal masses. Neurol India 55:267-273, 2007

29. Saini J, Gupta AK, Jolapara MB, Chatterjee S, Pendharkar HS, Kesavadas C, et al: Imaging findings in intracranial aspergillus infection in immunocompetent patients. World Neurosurg 74:661-670, 2010

30. Salaki JS, Louria DB, Chmel H: Fungal and yeast infections of the central nervous system. A clinical review. Medicine (Baltimore) 63:108-132, 1984

31. Selvam M, Pande A, Chakravarthy VM, Ramamurthi R: Invasive rhino-cerebral fungal granuloma. Neurol India 58:270-276, 2010

32. Sharma BS, Khosla VK, Kak VK, Banerjee AK, Vasishtha RK, Prasad KS, et al: Intracranial fungal granuloma. Surg Neurol 47:489-497, 1997

33. Sharma RR, Gurusinghe NT, Lynch PG: Cerebral infarction due to Aspergillus arteritis following glioma surgery. Br J Neurosurg 6:485-490, 1992

34. Siddiqui AA, Bashir SH, Ali Shah A, Sajjad Z, Ahmed N, Jooma R, et al: Diagnostic MR imaging features of craniocerebral Aspergillosis of sino-nasal origin in immunocompetent patients. Acta Neurochir (Wien) 148:155-166, 2006

35. Siddiqui AA, Shah AA, Bashir SH: Craniocerebral aspergillosis of sinonasal origin in immunocompetent patients: clinical spectrum and outcome in 25 cases. Neurosurgery 55:602-613, 2004
36. Sundaram C, Umabala P, Laxmi V, Purohit AK, Prasad VSSV, Panigrahi M, et al: Pathology of fungal infections of the central nervous system: 17 years' experience from Southern India. Histopathology 49:396-405, 2006

37. Uppar A, Raj ARP, Konar S, Kandregula S, Shukla D, Somanna S, et al: Intracranial cryptococcoma-clinicopathologic correlation and surgical outcome: a single-institution experience. World Neurosurg 115:e349-e359, 2018

38. Walsh TJ, Hier DB, Caplan LR: Fungal infections of the central nervous system: comparative analysis of risk factors and clinical signs in 57 patients. Neurology 35:1654-1657, 1985

39. Yanai Y, Wakao T, Fukamachi A, Kunimine H: Intracranial granuloma caused by Aspergillus fumigatus. Surg Neurol 23:597-604, 1985

40. Young RF, Gade G, Grinnell V: Surgical treatment for fungal infections in the central nervous system. J Neurosurg 63:371-381, 1985

\section{Disclosures}

The authors report no conflict of interest concerning the materials or methods used in this study or the findings specified in this paper.

\section{Author Contributions}

Conception and design: all authors. Acquisition of data: Prabhuraj, Mishra, Nandeesh, Chandrashekar. Analysis and interpretation of data: Prabhuraj, Mishra, Shukla, Ramalingaiah, Arivazhagan, Bhat, Somanna, Devi. Drafting the article: Prabhuraj, Mishra, Nandeesh, Chandrashekar, Arivazhagan, Devi. Critically revising the article: Prabhuraj, Mishra, Shukla, Chandrashekar, Ramalingaiah, Arivazhagan, Bhat, Somanna, Devi. Reviewed submitted version of manuscript: all authors. Approved the final version of the manuscript on behalf of all authors: Prabhuraj. Statistical analysis: Prabhuraj, Mishra, Shukla.

\section{Correspondence}

Andiperumal Raj Prabhuraj: National Institute of Mental Health and Neurosciences, Karnataka, India.drprabhuraj@yahoo.co.in. 